\title{
»IMMER DIE GLEICHEN KLASSIKER!« HeAVy METAL UND deR TRADITIONSSTROM
}

\section{Dietmar Elflein}

Eine Diskussion über Kanonbildungen in der populären Musik ist immer auch eine Diskussion über Fragen der Erinnerung. Die Forschung zu Gedächtnis und Erinnerung kennt neben dem Kanon jedoch noch weitere Konzepte der Aufbewahrung dessen, 'was bleibt < - so zum Beispiel den Traditionsstrom, ein Konzept, das auf den Assyrologen Leo Oppenheim zurückgeht (vgl. Assmann 2000: 55). Wie und warum sich der Traditionsstrom von einem wie auch immer definierten Kanon ${ }^{1}$ unterscheidet, möchte ich im ersten Teil dieses Artikels diskutieren, im zweiten Teil soll dann für den Musikstil Heavy Metal ein Zugang zum Traditionsstrom gesucht werden.

\section{Traditionsstrom vs. Kanon}

Mit relativ großer Sicherheit wird ein Musikstück wie »The Number Of The Beast « von Iron Maiden von sehr vielen Menschen mit einer gewissen popularmusikalischen Vorbildung mit Heavy Metal in Verbindung gebracht. Diese Assoziation geschieht aufgrund von Erinnerungen an ähnliche bereits gehörte Musiken und deren Bezeichnung. Individuelles Erinnern wird zu einer sich in der Gegenwart vollziehenden Operation des Zusammenstellens jeweils verfügbarer Daten. Für die neurologische Hirnforschung ist dabei »jede Reaktivierung einer Gedächtnisspur zugleich eine Neueinschreibung [...], die die Ersterfahrung notwendig überformt « (Assmann 2005: 107).

Damit lässt sich - neben der Gegenwartsbezogenheit - ein zweites zentrales Merkmal des Erinnerns anführen: sein konstruktiver Charakter. Erinnern wird wie sein Gegenteil, das Vergessen, als ein Prozess, Erinnerung als dessen Ergebnis und Gedächtnis als eine Fähigkeit oder eine verän-

1 In der angelsächsischen Musikwissenschaft wird das Für und Wider der Kanonisierung bereits seit Längerem diskutiert; vgl. etwa Kerman 1983, Bergeron/ Bohlmann 1992, Everist 1999, Webber 1999. 
derliche Struktur konzipiert. Individuelle und kollektive Erinnerung ist nie ein Spiegel einer authentischen Vergangenheit, wohl aber ein aussagekräftiges Indiz für die Bedürfnisse und Belange der Erinnernden in der Gegenwart. Das Gedächtnis wiederum ist eine soziale und individuelle Konstruktion zur Selbstvergewisserung der eigenen Geschichte, des eigenen Standpunkts. Wer bin ich und wo stehe ich (vgl. Erll 2005: 1-13)? Abstraktionen wie die Genre- und Stilbildung in der Musik - Iron Maiden spielen Heavy Metal dienen der Organisation und der Kommunizierbarkeit dieser Selbstvergewisserung und greifen zu diesem Zweck auf den Traditionsstrom zu. Der Traditionsstrom ist in dieser Argumentation eine Metapher für ein Netzwerk, in dem ein Teil aller ein bestimmtes Thema betreffenden Daten verfügbar gehalten wird.

Der bisher implizierte fließende Übergang von individuellen zu sozialen bzw. kollektiven Gedächtnisformen ist in der wissenschaftlichen Diskussion nicht selbstverständlich. So arbeitet die neurologische Hirnforschung eigentlich ausschließlich am Gedächtnis jeweils eines konkreten Individuums, dessen Langzeitgedächtnis nach beispielsweise Markowitsch/Welzer (2005: 206) in fünf unterschiedliche Gedächtnissysteme ${ }^{2}$ mit unterschiedlichen Zuständigkeiten unterteilt wird, deren Wirken dem Individuum nur zum Teil bewusst wird. ${ }^{3}$ Harald Welzer (2005: 111; 2002: 144) schlägt als verbindendes Element ein bereits sozial verfasstes autobiographisches Gedächtnis vor, das eine den fünf Gedächtnissystemen übergeordnete Struktur bildet. Aleida Assmann spricht von der gegenseitigen Abhängigkeit der drei Dimensionen (neuronal, sozial, kulturell) des Gedächtnisses:

»Während im Prozess des Erinnerns in der Regel alle drei Dimensionen: der neuronalen Strukturen, der sozialen Interaktion und der symbolischen Medien involviert sind, unterscheiden sich die unterschiedlichen Ebenen des Gedächtnisses darin, dass hier jeweils ein anderer Aspekt im Mittelpunkt steht« (Assmann 2006: 31f).

Das sozial verfasste autobiographische Gedächtnis partizipiert also über den Zugriff auf symbolische Medien an mindestens zwei sozialen und kollektiven

2 Episodisches, semantisches, perzeptuelles, prozedurales und das unübersetzbar Priming genannte Gedächtnissystem. Zur Definition von Gedächtnissystemen siehe z.B. Tulving 2005: 56.

3 Bewusste Gedächtnisinhalte finden sich im episodischen oder semantischen Gedächtnis. Unbewusste Gedächtnisinhalte, die uns beispielsweise Auto fahren lassen, ohne uns an jede einzelne der dafür notwendigen Handlungen bewusst erinnern zu müssen, liegen im impliziten Gedächtnis, das sich aus dem Priming genannten und dem prozeduralen Gedächtnissystemen zusammensetzt. Eine Zwischenstufe nimmt das perzeptuelle Gedächtnissystem ein, das dem Wiedererkennen von Reizen dient (vgl. Welzer 2002: 25-30). 
Gedächtnisformen ${ }^{4}$, die definiert werden müssen, um das Konzept des Traditionsstroms zu verstehen: das kulturelle und das kommunikative Gedächtnis. Jan Assmann, der den Begriff des kulturellen Gedächtnisses wieder und erfolgreich in die wissenschaftliche Diskussion eingebracht hat, definiert das kulturelle Gedächtnis als den

»jeder Gesellschaft und jeder Epoche eigentümliche[n] Bestand an Wiedergebrauchs-Texten, -Bildern und -Riten [...], in deren >Pflege « sie ihr Selbstbild stabilisiert und vermittelt, ein kollektiv geteiltes Wissen vorzugsweise (aber nicht ausschließlich) über die Vergangenheit, auf das eine Gruppe ihr Bewusstsein und ihre Eigenart stützt« (Assmann 1988: 15).

Das kommunikative Gedächtnis, das Welzer (2001: 13) und Aleida Assmann (2006: 26) als »Kurzzeitgedächtnis der Gesellschaft « bezeichnen, ist dagegen laut Jan Assmann (1988: 10) durch ein hohes Maß an Ungeformtheit, Beliebigkeit und Unorganisiertheit gekennzeichnet:

»Es lebt in interaktiver Praxis im Spannungsfeld der Vergegenwärtigung von Vergangenem durch Individuen und Gruppen. Es ist an die Existenz der lebendigen Träger und Kommunikatoren von Erfahrung gebunden und umfasst etwa 80 Jahre, also drei bis vier Generationen. Der Zeithorizont des >kommunikativen Gedächtnisses` wandert entsprechend >mit dem fortschreitenden Gegenwartspunkt mit« (Welzer 2001: 13).

Interessant ist der Moment des Überganges zwischen diesen beiden Gedächtnisformen - das etwa 80 Jahre zurückliegende »floating gap«, wie es der Ethnologe Jan Vansina in seiner Arbeit über Oral History (Vansina 1985) benennt. Laut Jan Assmann (1992: 51) ist sogar bereits die Hälfte dieses das kommunikative Gedächtnis eingrenzenden Zeitraumes, also circa 40 Jahre, eine kritische Schwelle für den Beginn eines Wunsches nach Historisierung. Wie wechselt also etwas vom an die relative Gegenwart gekoppelten kommunikativen Gedächtnis ins mythische kulturelle Gedächtnis - wobei die Grenze zwischen den beiden Formen der Erinnerung sowohl scharf als auch fließend gedacht werden kann? Wie entsteht im Rahmen dieses theoretischen Modells eine historisierbare Vergangenheit mit einer im Gegensatz zur mythischen Urzeit wachsenden Distanz zur Gegenwart? Hier kommt der Traditionsstrom ins Spiel, der »die zum Wiedergebrauch bestimmten Texte aufnimmt « (Assmann 2000: 55). Jan Assmann betrachtet den Traditionsstrom als einen historischen Vorläufer des Kanons. Damit rückt dieser näher ans kommunikative Gedächtnis als ein zwangsläufig zum kulturellen Gedächtnis

4 Jan Assmann (1992: 20) spricht von vier Außendimensionen des Gedächtnisses, neben dem kulturellen und dem kommunikativen Gedächtnis sind dies das »mimetische Gedächtnis « und das »Gedächtnis der Dinge«. 
gehöriger Kanon. Trotzdem besteht der Traditionsstrom aber bereits aus kulturellen Artefakten, aus Texten normativen und formativen Anspruchs, die nicht aus der mündlichen Überlieferung stammen, sondern quasi originär geschrieben wurden. Der Traditionsstrom begleitet die Formierung eines überzeitlichen kulturellen Gedächtnisses - ein Aspekt, der dieses Konzept für die Beschäftigung mit populärer Musik und ihrer relativ kurzen Geschichte, die erst kürzlich den kritischen Punkt der Hälfte des »floating gap« überschritten hat, attraktiv macht.

»Dieser Traditionsstrom ist ein lebendiger Fluss: Er verlagert sein Bett und führt bald mehr, bald weniger Wasser. Texte geraten in Vergessenheit, andere kommen hinzu, sie werden erweitert, abgekürzt, umgeschrieben, katalogisiert in wechselnden Zusammenstellungen « (Assmann 1992: 92).

Dieses Zitat umreißt sehr schön den Umgang mit Musik innerhalb einer bestimmten Szene oder innerhalb eines Genres. Manche Stücke werden vergessen, neue kommen hinzu. Sie werden umgeschrieben (gecovert), bearbeitet und anthologisiert in einem nicht endenden Strom von persönlichen Plattensammlungen, Mixtapes (-CDs) und iPod-Playlists. Die Musikindustrie trägt aktiv zum Vergessen bei, indem der Bestand zugänglicher, also erwerbbarer Stücke klein gehalten wird, während permanent Neues veröffentlicht wird, das seinen Platz im Traditionsstrom finden soll. Durch zahllose Best of-Veröffentlichungen und thematische Zusammenstellungen versucht die Musikindustrie einen bestimmten Bereich des Traditionsstroms als endgültig normativ und formativ darzustellen. Kleinstfirmen, Plattensammler, private Webseitenbetreiber, Blogs und die illegalisierten Filesharing-Netzwerke entreißen permanent Stücke diesem Vergessen und halten sie weiter zugänglich für Interessierte und Neugierige. In diesem ungleichen Wechselspiel prägen sich Strukturen von Peripherie und Zentrum, von fast vergessenen und viel kopierten bzw. bearbeiteten Texten heraus, die weiter im Fluss sind.

Ich begreife den Traditionsstrom dementsprechend als Diskurs, wie er beispielsweise von Siegfried Jäger (2006: 84) in einem analogen Bild als »Fluss von Wissen bzw. sozialen Wissensvorräten durch die Zeit« beschrieben wird (Jäger 2006: 84). Der Traditionsstrom fließt also nicht nur durch die Geschichte, er wird gleichzeitig in sie hinein projiziert - mit bestimmten Absichten und aufgrund bestimmter gegenwärtiger Erfahrungen, die eine bestimmte Vergangenheit benötigen, damit sie bewältigbar bleiben. Der Traditionsstrom beinhaltet damit eine sich verändernde Auswahl dessen, was gewusst werden kann, darf und soll. Er entspricht weder dem gesamten verfügbaren Wissen noch dem gesamten Wissen, sondern dem 
momentan nicht vergessenen Teil des verfügbaren Wissens. Diese feine Unterscheidung zwischen dem gesamten verfügbaren Wissen und dem gesamten Wissen bedarf einer kurzen Erläuterung. Begreift man im Sinne Michel Foucaults Diskurs als die Gesamtheit der Regeln, die einer sprachlichen Praxis immanent sind, so definieren die Regeln des Diskurses für einen bestimmten Zusammenhang oder ein bestimmtes Wissensgebiet, was sagbar ist, was gesagt werden soll, was nicht gesagt werden darf und welcher Sprecher was wann sagen darf. Der einen Diskurs Beschreibende oder Beobachtende ist dabei immer auch Teil des Diskurses.

»Die Untersuchung der > ̈̈ußerungsmodalitäten $<$ ( ‘wer spricht $<$ ), der >Formation der Begriffe und Strategien usw. soll die Selektion, Kanalisierung, die Organisation und Kontrolle: das >Regime des Diskurses aufweisen, und das heißt zugleich: soll die diskontinuierlich auftauchenden Ereignisse in ihrer Streuung beschreiben « (Fohrmann 1997: 373).

Die Idee der Verfügbarkeit des gesamten Wissens wird so zu einer rein theoretischen Möglichkeit, die in der diskursiven Praxis als Ideologie erscheint. Die diskursive Regelungsinstanz, die die Möglichkeit des Zugriffs sowohl auf den vergessenen Teil des verfügbaren Wissens als auch auf den nicht verfügbaren Teil des gesamten Wissens regelt, hat Foucault in seiner Archäologie des Wissens "Archiv« genannt - das "Gesetz dessen, was gesagt werden kann, das System, das das Erscheinen der Aussagen als einzelne Ereignisse beherrscht« (Foucault 1973: 187).

Mit der Einführung der Veränderlichkeit in das Konzept des Kanons, die unter anderem William Webber für den musikalischen Kanon ${ }^{5}$ anregt, verschwindet scheinbar die Notwendigkeit, zwischen den Konzepten des Traditionsstroms, der »die zum Wiedergebrauch bestimmten Texte aufnimmt « (Assmann 2000: 55), und dem Kanon als »Stillstellung des Traditionsstroms« (ebd.: 143) zu differenzieren. Allerdings bleibt der Kanon - im Unterschied zum Traditionsstrom - weiterhin ausschließlich Teil des kulturellen Gedächtnisses. So unterteilt Aleida Assmann das kulturelle Gedächtnis in das als Vordergrund oder aktiv zu denkende Funktions- und das als Hintergrund oder passiv zu denkende Speichergedächtnis - ersteres entspricht dem Kanon, letzteres dem Archiv. ${ }^{6}$ Unklar bleibt in diesem Konzept jedoch

5 Webber (1999) identifiziert drei Typen des musikalischen Kanons: den wissenschaftlichen, den pädagogischen und den performativen Kanon. Als formative Aspekte der drei Kanontypen benennt er Handwerk, Repertoire, Kritik und Ideologie.

6 Aleida Assmanns »Archiv « unterscheidet sich grundlegend von Foucaults »Archiv«, da es auf einem transzendentalpragmatischen Diskursbegriff nach Habermas beruht, der im Gegensatz zu Foucault die Möglichkeit eines herrschaftsfreien Diskurses benötigt (vgl. Assmann 1999: 132-142 und 2006: 54-58). 
wiederum die Art und Weise des Übergangs zwischen Funktions- und Speichergedächtnis. ${ }^{7}$ Wie ist beispielsweise der Weg des dekanonisierten Wissens ins Archiv? Oder existiert gar kein derartiger Weg, ist etwas grundsätzlich entweder shier o oder >dort -, kanonisiert oder archiviert? Das dem Kanonkonzept inhärente Moment der Unbeweglichkeit, welches Jan Assmann das Bild der "Stillstellung des Traditionsstroms « benutzen und Katherine Bergeron und Philip V. Bohlman (1992) einen von ihnen herausgegebenen Sammelband Disciplining Music nennen ließ, bleibt damit trotz der Einführung seiner Veränderlichkeit erhalten.

Ein Kanon fließt nicht. Die Beweglichkeit des Traditionsstroms ist dagegen unstrittig und unproblematisch. Er fließt im Gegensatz zum Speichergedächtnis nicht nur, aber auch im Vordergrund. Er bietet zudem die Möglichkeit, sowohl kommunikatives und kulturelles Gedächtnis als auch Funktions- und Speichergedächtnis zu verbinden, und kann damit auch das Dekanonisierte transportieren und beherbergen. Des Weiteren kann er als Metapher für die Möglichkeit diskursiver Veränderung nach den jeweils aktuellen Maßgaben des Archivs verwendet werden. All dies führt mich zu einer Bevorzugung des Konzepts des Traditionsstroms gegenüber dem des Kanons - wohl wissend, dass auch der Begriff der Tradition uneindeutig ist.

»Der Begriff der Tradition hat zwei Bedeutungen: wenn wir ihn vom Standpunkt von Gedächtnis und Erinnerung aus betrachten, [...] dann erscheint er als der Gegensatz zum Gelebten, Verkörperten und Kommunizierten und als der Inbegriff des in symbolischen Formen ausgelagerten und von Institutionen verwalteten Wissens. Wenn wir ihn dagegen vom Standpunkt der Schrift aus betrachten wie in der jüdischen und katholischen Tradition, dann erscheint er als der Gegensatz des schriftlich fixierten und der Inbegriff des an lebendige Träger gebundenen, inkarnierten Wissens« (Assmann 2000: 81).

Aus der Perspektive des kommunikativen Gedächtnisses erscheint der Traditionsstrom damit als fest gefügt, aus der Perspektive des kulturellen Gedächtnisses dagegen als lebendig und veränderlich.

Wenn das Gedächtnis eine soziale und individuelle Konstruktion zur Selbstvergewisserung der eigenen Geschichte, des eigenen Standpunkts ist, das auf den Traditionsstrom als Reservoir des momentan verfügbaren Wissens zugreift, so geschieht dieser Zugriff auch in Form von Erwartungen und Modellen oder - mit dem russischen Literaturwissenschaftler Mikhail Bakhtin (1986: 68) gesprochen - in Form der vorwegnehmenden Imagination

7 Die bereits thematisierte Frage des Übergangs zwischen kommunikativem und kulturellem Gedächtnis wird quasi in das theoretische Konstrukt des kulturellen Gedächtnisses hinein verlagert. 
einer Äußerung mittels ihrer Eingebundenheit in Genres - bei Bakhtin »speech genres« - mit jeweils bestimmten Regeln und Formen.

Ein Begriff wie Heavy Metal produziert damit bestimmte auf Erfahrung beruhende Erwartungen, wie eine derartige Äußerung bzw. Musik zu klingen habe, an denen die konkrete Äußerung wiederum gemessen und mit denen sie verglichen wird. Es existiert also sowohl ein Erwartungshorizont beim Rezipienten als auch bestimmte Kompositionsmodelle beim Produzenten, wenn er oder sie ein Heavy Metal-Stück produzieren will. Coverversionen wie die deutsche Version der Black Sabbath-Komposition "Paranoid« - der deutsche Schlager »Der Hund von Baskerville« von Cindy \& Bert aus dem Jahr 1971 - verdeutlichen, dass sich der durch das die Aufnahme eröffnende Gitarrenriff bestimmte Erwartungshorizont des Rezipienten und das Kompositionsmodell des Produzenten auf ein gleiches Reservoir an Vorbildern im Traditionsstrom - Black Sabbath - beziehen können, ohne daraus zwangsläufig die gleichen Schlussfolgerungen zu ziehen. Erwartungen können enttäuscht, Kompositionsmodelle verändert werden.

Es entsteht nach Hans Robert Jauß, der den Erwartungshorizont bereits in den 1960er Jahren in die literaturwissenschaftliche Diskussion eingeführt hat, ein dialogisch-prozesshaftes Verhältnis von Werk, Publikum und weiterem Werk. ${ }^{8}$ Der Traditionsstrom wird zur endlosen Kette musikalischer Äußerungen. Da sprachliche Äußerungen für Bakhtin über ihre Abgeschlossenheit und Aufforderung zur Reaktion definiert sind, existieren diese nur als Teil eines Dialoges bzw. als Teil eines Netzwerkes bzw. einer Kette von Äußerungen (vgl. Bakhtin 1986: 76). Diese Kette ist endlos, weil laut Bakhtin jeder Sprecher, also Produzent von Sprache, gleichzeitig immer auch Rezipient ist, also auf eine vorhergehende Äußerung reagiert: "Any speaker is himself a respondent to a greater or lesser degree. He is not, after all, the first speaker, the one who disturbs the eternal silence of the universe « (ebd.: 69).

Die Kette der Äußerungen hebt die Trennung von Produktion und Rezeption auf. Gleichzeitig bleibt der Autor als Produzent von Sprechakten immer anwesend, er markiert die jeweilige Perspektive des Blickes auf das Netzwerk. In einer poststruktural-diskursiven Vorstellung bleibt die zentrale Frage des 'wer spricht < erhalten. Ein Blick auf den Traditionsstrom beinhal-

8 »Denn erst durch seine [gemeint ist der Adressat oder Leser, D.E.] Vermittlung tritt das Werk in den sich wandelnden Erfahrungshorizont einer Kontinuität [...]. Die Geschichtlichkeit der Literatur wie ihr kommunikativer Charakter setzen ein dialogisches und zugleich prozeßhaftes Verständnis von Werk, Publikum und neuem Werk voraus« (Jauß 1979: 169). 
tet damit eine jeweils momentane und von unterschiedlichen Interessen beeinflusste Auswahl aus der endlosen Kette der Äußerungen.

\section{Der Traditionsstrom am Beispiel von Heavy Metal}

Wie ist nun ein Zugang zu einem so verstandenen Traditionsstrom möglich zu musikalischen Äußerungen, denen im Moment ein normativer und formativer Anspruch für den Musikstil zugebilligt wird, zu Bands ${ }^{9}$, deren Gesamtwerk - oder Teile davon - den Erwartungshorizont prägen und als Anregungen für Kompositionsmodelle dienen. Da dieser Prozess im Fluss ist, ist der Blick auf ein einzelnes Ereignis, z.B. das Programm eines Open Air Festivals, das Inhaltsverzeichnis einer Fachzeitschrift oder auch die persönlichen Erinnerungen und Vorlieben des Forschers, nicht ausreichend, um die Dynamik zu begreifen. Vielmehr müssen unterschiedliche Quellen genutzt werden. Eine derartige Möglichkeit besteht im Vergleich von Auswahllisten aus dem Traditionsstrom des Heavy Metal - also von Listen mit Titeln wie »Die 500 besten Heavy Metal-Songs aller Zeiten« etc. pp. -, ohne die Unterschiedlichkeit der Quellen zu problematisieren, sondern als bewusst heterogene Datensammlung, die ein temporäres Abbild des Prozesses der Formierung eines kulturellen Gedächtnisses bieten soll.

Die Auswertung einer derartigen Stichprobe im Rahmen meiner Dissertation zur musikalischen Sprache des Heavy Metal zeigt eindeutige Präferenzen bezüglich wichtig erachteter Bands bzw. einzelner oder Gruppen von Tonträgern, die im Folgenden nur schlaglichtartig dargestellt werden können. ${ }^{10}$ Grundlage für die Datenerhebung sind:

(i) sechs Hörempfehlungen in wissenschaftlicher und populärwissenschaftlicher Literatur: Christe 2003, Popoff 2003, Schäfer 2001, Udo 2002, Walser 1993, Weinstein 1991;

(ii) Listen aus zwei deutsch- und drei englischsprachigen Fachzeitschriften: Hitparader (USA; 1982, 1989), Kerrang! (UK; 1989, 1998, 1999, 2002),

9 Unter ihrem Eigennamen firmierende Musiker wie beispielsweise Ted Nugent, Ozzy Osbourne oder David Lee Roth werden in der Folge der Einfachheit halber unter dem Begriff Band subsumiert.

10 Stichtag ist der 30.5.2006 - für die Webseiten gilt dies auch als Zugriffsdatum. Die Stichprobe dient im Rahmen der Dissertation als Grundlage für die Auswahl der zu untersuchenden Musik. 
Metal Hammer (BRD; 2005), Rock Hard (BRD; 2005), Terrorizer (UK; nicht datiert $\left.{ }^{11}\right)$;

(iii) zwölf Webseiten: angelfire.com, anus.com, bleeding4metal.net, heavymetal-heaven.de, metal-bite.com, metal-dungeon.de, metal-rules. com, musicline.de/de/genre/lexikon/metal, pommesgabel.de, rocklist. net, ruthlessreviews.com, vampster.com.

Diese 23 Quellen bieten 68 Listen $^{12}$ mit jeweils unterschiedlichem inhaltlichen Schwerpunkt an, die alle um Tonträger zentriert sind. Gegenüber Listen, die auf Bands zentriert sind, haben diese den Vorteil, dass nicht automatisch das Gesamtwerk der Bands in den Blick kommt, sondern ein differenzierterer Blick möglich ist. Um die Vergleichbarkeit der Listen zu gewährleisten, mussten einige Anpassungen vorgenommen werden. Da drei Listen einzelne Songs beinhalten, alle anderen hingegen Alben, wurden die Songs den jeweiligen Alben zugeschrieben. Zudem sind manche Listen alphabetisch, andere dagegen hierarchisch aufgebaut. Daraus folgt einerseits, dass die Listen nicht sinnvoll auf eine gemeinsame Länge zu kürzen sind - die Listen umfassen zwischen acht und 500 Einträge - und andererseits auch eine potentielle Rangfolge nicht ausgewertet werden kann.

Die Stichprobe umfasst insgesamt 821 Bands aus 27 Staaten mit 1526 Tonträgern aus 41 Jahren (1966-2007). Ausgewertet wurde das Vorkommen einer Band oder eines Tonträgers sowohl in den 68 Listen als auch als Korrektiv in den 23 Quellen.

In der Folge sollen einige der Ergebnisse kurz skizziert werden. Die Auswertung der nationalen Herkunft der erfassten Bands zeigt, dass 411 von 821 und damit ziemlich genau $50 \%$ aus den USA stammen. Es folgen Großbritannien mit fast $19 \%$ (155), die BRD ${ }^{13}$ mit knapp $8 \%$ (65) und Schweden mit ca. $6 \%$ (49). Kanada (24), Finnland (22) und Norwegen (22) bewegen sich zwischen $2 \%$ und $3 \%$. Zwischen $1 \%$ und $2 \%$ liegen die Niederlande (15) und Dänemark (9). Alle anderen Herkunftsnationen kommen auf weniger als $1 \%$ der Gesamtzahl. ${ }^{14}$

11 Die Quelle für die Terrorizer-Listen ist die Webseite www.rocklist.net (Zugriff am 30.5.2006).

12 Eingang in die Stichprobe fanden nur Listen mit überzeitlichem Anspruch. Ausgeschlossen waren damit so genannte Jahrescharts und sonstige Momentaufnahmen auf zeitlicher Basis.

13 Es wurde keine Unterscheidung zwischen BRD (alt) und DDR gemacht. Alle bereits vor der Wiedervereinigung aktiven genannten Bands stammen aus Westdeutschland.

14 Japan und Schweiz (je 7 Nennungen), Australien und Frankreich (je 5), Brasilien (4), Italien und Polen (je 3), Belgien und Ungarn (je 2), sowie Griechenland, 
Heavy Metal stellt sich damit in der Stichprobe als musikalisches Genre mit eindeutig angelsächsischer Dominanz dar. Auf den Plätzen folgen wahlweise die BRD oder Skandinavien. ${ }^{15}$ Allerdings stammen immerhin $27 \%$ (222) der erfassten Bands nicht aus dem angelsächsischen Raum. Zieht man von diesen - aufgrund einer möglichen Überrepräsentation deutscher Bands in einer in Deutschland erstellten Stichprobe - die deutschen Bands ab, so verbleiben $19 \%$ (157) der Bands. Von diesen stammen jedoch wiederum nur $1,46 \%$ (12) nicht aus Europa.

Vergleicht man dieses Ergebnis mit Listen aus stilistisch anders ausgerichteten Magazinen zur populären Musik wie Rolling Stone, Visions oder Spex, so zeigt sich in der Heavy Metal-Stichprobe ein deutlich größerer Anteil nicht-angelsächsischer Bands. ${ }^{16}$ Dieser verschwindet jedoch, wenn man sich auf die konsensfähigen Bands konzentriert.

\begin{tabular}{|l|cccc|}
\hline & Staat & $\begin{array}{c}\text { Nennungen } \\
\text { in Listen }\end{array}$ & $\begin{array}{c}\text { Nennungen } \\
\text { in Quellen }\end{array}$ & $\begin{array}{c}\text { Anzahl der ge- } \\
\text { nannten Veröf- } \\
\text { fentlichungen }\end{array}$ \\
\hline Metallica & USA & 70 & 20 & 9 \\
Judas Priest & GB & 70 & 17 & 13 \\
Black Sabbath & GB & 69 & 14 & 14 \\
Iron Maiden & GB & 66 & 14 & 10 \\
Slayer & USA & 47 & 15 & 7 \\
Megadeth & USA & 39 & 18 & 7 \\
AC/DC & AUS & 55 & 14 & 11 \\
Deep Purple & GB & 32 & 15 & 7 \\
Led Zeppelin & GB & 44 & 13 & 8 \\
Motörhead & GB & 39 & 13 & 10 \\
\hline
\end{tabular}

Tabelle 1: Die zehn meistgenannten Bands

Irland, Island, Österreich, Portugal, Spanien, Südafrika und Tschechien mit je einer Nennung. Drei Bands sind nicht eindeutig zuzuordnen.

15 Dänemark, Finnland, Island, Norwegen und Schweden erreichen zusammen 103 Nennungen.

16 Von den in den $\$ 500$ besten Alben aller Zeiten « der deutschen Ausgabe des Rolling Stone (2004) vertretenen 285 verschiedenen Bands sind nur 9,2\% nichtangelsächsischer Herkunft, bei den $» 100+17$ besten Platten aller Zeiten « aus Spex (2000) 9,6\% und den »150 Records For Eternity « aus Visions (2005) 15,5\%. Zieht man ebenfalls jeweils die deutschen Bands ab, so verbleiben 3,2\% (Rolling Stone), 4,8\% (Spex) und 8,8\% (Visions). 
Die aus der Synthese der Auswertungen nach Nennungen in Listen und in Quellen erstellte Meta-Liste der zehn meistgenannten Bands verzeichnet ausnahmslos Bands aus dem angelsächsischen Raum. Eine reine Auswertung nach Listen platziert AC/DC mit 55 Nennungen vor Slayer und Megadeth, bei einer Auswertung nach Quellen liegen Megadeth dagegen mit 18 Nennungen direkt hinter den in beiden Kategorien führenden Metallica. Das Sample dieser zehn Bands bildet eine eindeutige Spitzengruppe.

Konkret werden nur siebzehn der insgesamt 821 Bands überhaupt in mehr als der Hälfte der 23 Quellen genannt. Bei den Nennungen in Listen zeigt sich ein analoges Bild. Nur 27 Bands werden mehr als zwanzig Mal in den 68 Listen genannt.

Schränkt man jedoch die stilistische Bandbreite auf Bands ein, die dem Extreme Metal zugeordnet werden können ${ }^{17}$, so ergibt sich eine Liste von Bands, deren nationale Herkunft keine angelsächsische Dominanz mehr zeigt.

\begin{tabular}{|l|cccc|}
\hline \multicolumn{1}{|c|}{ Band } & Staat & $\begin{array}{c}\text { Nennungen } \\
\text { in Listen }\end{array}$ & $\begin{array}{c}\text { Nennungen } \\
\text { in Quellen }\end{array}$ & $\begin{array}{c}\text { Anzahl der ge- } \\
\text { nannten Veröf- } \\
\text { fentlichungen }\end{array}$ \\
\hline Sepultura & BRA & 29 & 13 & 7 \\
Death & USA & 22 & 12 & 5 \\
Celtic Frost & CH & 20 & 11 & 3 \\
Morbid Angel & USA & 20 & 9 & 6 \\
Bathory & SWE & 14 & 11 & 4 \\
Emperor & NOR & 14 & 10 & 3 \\
Carcass & GB & 18 & 9 & 4 \\
Napalm Death & GB & 14 & 8 & 4 \\
Entombed & SWE & 12 & 9 & 3 \\
Dimmu Borgir & NOR & 12 & 9 & 4 \\
\hline
\end{tabular}

Tabelle 2: Die zehn meistgenannten Extreme Metal-Bands

Nur noch vier der zehn meistgenannten Extreme Metal-Bands stammen aus dem angelsächsischen Raum. Allerdings ist die Anzahl der Nennungen für die Extreme Metal-Bands insgesamt erheblich geringer als bei den zehn meistgenannten Bands. Nur Sepultura bewegen sich aufgrund der Nennung in 13 Quellen in der Nähe einer Aufnahme in diese Meta-Liste. Möglicherweise ist

17 Die Zuordnung erfolgt nach Keith Kahn-Harris (2007). 
die weltweite Verbreitung und Wahrnehmung von Heavy Metal also vor allem seinen extremeren Spielarten geschuldet. Allerdings sind die erhobenen Daten nicht ausreichend, um hier fundierte Aussagen zu treffen.

Betrachtet man die herausgehobenen Alben der Auswertung, so zeigt sich zum einen erneut eine eindeutig angelsächsische Dominanz.

\begin{tabular}{|l|lccc|}
\hline \multicolumn{1}{|c|}{ Band } & \multicolumn{1}{|c}{ Album } & Jahr & Staat & $\begin{array}{c}\text { Nennungen in } \\
\text { Listen/Quellen }\end{array}$ \\
\hline AC/DC & Back In Black & 1980 & AUS & $16 / 12$ \\
Guns N' Roses & Appetite For Destruction & 1987 & USA & $16 / 12$ \\
Metallica & Kill 'em All & 1983 & USA & $15 / 14$ \\
Metallica & Ride The Lightning & 1984 & USA & $15 / 13$ \\
Metallica & Master Of Puppets & 1986 & USA & $15 / 13$ \\
Slayer & Reign In Blood & 1986 & USA & $15 / 13$ \\
Black Sabbath & Paranoid & 1970 & GB & $15 / 11$ \\
Queensrÿche & Operation: Mindcrime & 1988 & CAN & $14 / 12$ \\
Led Zeppelin & IV & 1971 & GB & $14 / 12$ \\
Iron Maiden & The Number Of The Beast & 1982 & GB & $14 / 11$ \\
\hline
\end{tabular}

Tabelle 3: Die zehn meistgenannten Alben

Zum anderen dominieren Metallica mit drei Veröffentlichungen unter den zehn meistgenannten Alben auch die Auswertung nach Alben. Mit Guns N' Roses und Queensrÿche werden außerdem zwei Bands genannt, die nicht unter den zehn meistgenannten Bands in Tabelle 1 vertreten sind. Hier liegt jeweils eine große Wertschätzung für ein einzelnes Album vor, während die übrigen Alben als unwichtiger erscheinen. Erstaunlicherweise fehlt in Tabelle 3 ein Tonträger von Judas Priest - immerhin jener Band, die gemeinsam mit Metallica die meisten Nennungen in Listen auf sich vereinigen konnte. Bei Judas Priest verteilt sich die Wertschätzung so gleichmäßig auf mehrere Alben, dass für sich genommen keines zu den zehn meistgenannten gehört. Die in der Stichprobe herausgehobenen Alben einer Band entstammen zudem immer bestimmten Schaffensphasen, die häufig identisch mit der Frühphase einer Band sind oder eine bestimmte Besetzung repräsentieren, die wiederum nicht vollständig sein muss. Bei Judas Priest interessieren z.B. nur Veröffentlichungen mit Rob Halford als Sänger. Die drei Metallica-Alben unter den zehn meistgenannten entsprechen sowohl den drei ersten LPs 
der Band als auch allen offiziellen Tonträgern Metallicas mit Bassist Cliff Burton.

Beide Spitzengruppen, die der Alben wie der Bands, distanzieren sich deutlich von der restlichen Stichprobe. Nur 17 der 1526 Alben werden in mehr als zehn Quellen genannt und nur 145 Alben werden mindestens zehnmal in den Listen genannt. Auffällig ist zudem, dass acht der zehn meistgenannten Alben aus den 1980er Jahren stammen. ${ }^{18}$ Die zeitliche Verteilung der Erscheinungsdaten aller Alben der Stichprobe zeigt allerdings, dass mit $52 \%$ die Mehrheit der erfassten Tonträger nicht in den 1980er Jahren, sondern zwischen 1990 und 2006 erschienen ist.

\begin{tabular}{|c|c|c|}
\hline Jahrzehnt & $\begin{array}{c}\text { Absolute } \\
\text { Zahl }\end{array}$ & $\%$ \\
\hline $1960-69$ & 21 & 1,38 \\
$1970-79$ & 172 & 11,27 \\
$1980-89$ & 538 & 35,26 \\
$1990-99$ & 553 & 36,29 \\
$2000-06$ & 242 & 15,86 \\
\hline
\end{tabular}

Tabelle 4: Zeitliche Verteilung der Erscheinungsdaten der erfassten Tonträger

In der Meta-Liste der Alben ist diese zeitliche Periode damit deutlich unterrepräsentiert. Ein Vergleich mit den meistgenannten Extreme Metal-Alben zeigt, das diese ebenfalls mehrheitlich in den 1980er Jahren erschienen sind, wenn auch eine Schwerpunktverschiebung in die zweite Hälfte der 1980er Jahre feststellbar ist (s. Tabelle 5).

Im Extreme Metal-Bereich findet sich also bei der zeitlichen Verteilung im Gegensatz zur nationalen Verteilung kein anderer Trend als in der gesamten Stichprobe.

18 Die für Heavy Metal anscheinend bestimmenden Jahre von 1980-89 sind übrigens laut Ralf von Appen und André Doehring (2006: 23) im - in ihren Worten »Kanon der Pop- und Rockalben« unterrepräsentiert. 


\begin{tabular}{|l|lllc|}
\hline \multicolumn{1}{|c|}{ Band } & \multicolumn{1}{|c}{ Album } & Jahr & Staat & $\begin{array}{c}\text { Nennungen in } \\
\text { Listen/Quellen }\end{array}$ \\
\hline Emperor & In The Nightside Eclipse & 1994 & NOR & 10 / 09 \\
Possessed & Seven Churches & 1985 & USA & 08 / 08 \\
Napalm Death & Scum & 1987 & GB & 09 / 07 \\
Celtic Frost & Morbid Tales & 1984 & CH & 07 / 07 \\
Morbid Angel & Altars Of Madness & 1989 & USA & 07 / 07 \\
Entombed & Left Hand Path & 1990 & SWE & 07 / 07 \\
Sepultura & Beneath The Remains & 1989 & BRA & 07 / 07 \\
Celtic Frost & To Mega Therion & 1985 & CH & 07 / 06 \\
Death & Scream Bloody Gore & 1987 & USA & 07 / 06 \\
Mayhem & De Mysteriis Dom Satanas & 1994 & NOR & 06 / 06 \\
\hline
\end{tabular}

Tabelle 5: Die zehn meistgenannten Extreme Metal-Alben

\section{Synthese}

Die Stichprobe zeigt eindeutig bestimmte, den musikstilistischen Erwartungshorizont des Heavy Metal prägende Bands und Tonträger, die jeweils mindestens fünfzehn bis zwanzig Jahre alt sein müssen, um den normativen und formativen Anspruch des Traditionsstroms mitzubestimmen. Die 1980er Jahre bilden damit im Moment das die musikalische Sprache des Heavy Metal definierende Jahrzehnt. Je näher man der Gegenwart des kommunikativen Gedächtnisses kommt, desto breiter wird das Feld der in der Stichprobe versammelten Tonträger. Es bleibt unklar, welche Rolle diese in der zukünftigen Gestaltung des Traditionsstroms spielen werden. Neben neueren Veröffentlichungen sind auch nicht-angelsächsische Bands und extreme Spielarten des Heavy Metal in den oberen Regionen der Meta-Listen unterrepräsentiert. Möglicherweise werden sich jedoch in zehn Jahren einige Alben aus den 1990ern durchgesetzt haben und die angelsächsische Dominanz wird vermutlich durch skandinavische Bands aufgelöst werden.

Mit Black Sabbath, Judas Priest, Iron Maiden und besonders Metallica kristallisieren sich vier herausgehobene Bands heraus, deren musikalischen Äußerungen bestimmter Schaffensphasen ein normativer und formativer Anspruch zugesprochen wird. Meist handelt es sich dabei um eine bestimmte Besetzung oder die Frühphase einer Band. Die besondere Wertschätzung, 
die einzelnen Alben weiterer Bands - AC/DCs Back In Black, Guns N' Roses' Appetite For Destruction oder Slayers Reign In Blood - entgegengebracht wird, muss als die kleinstmögliche wertschätzbare Schaffensphase einer Band betrachtet werden. Das Gesamtwerk spielt dagegen bei keiner Band eine herausragende Rolle. Es erscheint somit sinnvoll, bei der Zusammensetzung der Stichprobe auf die Darstellbarkeit dieser Schaffensphasen zu achten und sich nicht unterschiedslos auf das Gesamtwerk einer Band einerseits oder ausschließlich einzelne Alben andererseits zu beschränken.

Das Konzept des Traditionsstroms erleichtert den Umgang mit derartigen Schaffensphasen, da er nur für einen normativen und formativen Anspruch, also eine Möglichkeit steht. Dagegen versucht ein Kanon jeweils einen Teilbereich des Traditionsstroms - und das heißt eben auch einzelne Alben - als endgültig normativ und formativ darzustellen. Ich bevorzuge deshalb das Konzept des Traditionsstroms gegenüber einer möglichen Differenzierung des Kanonbegriffs - auch auf Grund der größeren Nähe des Traditionsstroms zum die jeweils letzten 80 Jahre umfassenden kommunikativen Gedächtnis und der Assoziationsmöglichkeit als ein in der Zeit fließendes, zentrumsloses Netzwerk musikalischer Äußerungen.

\section{Literatur}

Appen, Ralf von / Doehring, André (2006). "Nevermind The Beatles, Here's Exile 61 and Nico: 'The Top 100 Records of All Time - a Canon of Pop and Rock Albums from a Sociological and an Aesthetic Perspective. In: Popular Music 25, H. 1, S. 21-39.

Assmann, Aleida (1999). Erinnerungsräume. Formen und Wandlungen des kulturellen Gedächtnisses. München: Beck.

Assmann, Aleida (2005). "Wie wahr sind unsere Erinnerungen?« In: Warum Menschen sich erinnern können. Fortschritte der interdisziplinären Gedächtnisforschung. Hg. v. Harald Welzer und Hans J. Markowitsch. Stuttgart: Klett-Cotta, S. 95-110.

Assmann, Aleida (2006). Der lange Schatten der Vergangenheit. Erinnerungskultur und Geschichtspolitik. München: Beck.

Assmann, Jan (1988). »Kollektives Gedächtnis und kulturelle Identität. In: Kultur und Gedächtnis. Hg. v. Jan Assmann und Tonio Hölscher. Frankfurt/M.: Suhrkamp, S. 9-19.

Assmann, Jan (1992). Das kulturelle Gedächtnis. Schrift, Erinnerung und politische Identität in frühen Hochkulturen. München: Beck.

Assmann, Jan (2000). Religion und kulturelles Gedächtnis. Zehn Studien. München: Beck.

Bakhtin, Mikhail (1986). Speech Genres and Other Late Essays. Hg. v. Carl Emerson und Michael Holquist. Austin: University of Texas Press.

Bergeron, Katherine / Bohlman, Philip V. (Hg.) (1992). Disciplining Music: Musicology and its Canons. Chicago: University of Chicago Press. 
Christe, Ian (2003). Sound of the Beast. The Complete Headbanging History of Heavy Metal. New York: Harper Collins.

Erll, Astrid (2005). Kollektives Gedächtnis und Erinnerungskulturen. Stuttgart, Weimar: Metzler.

Everist, Mark (1999). »Reception Theories, Canonic Discourses and Musical Value.« In: Rethinking Music. Hg. v. Nicholas Cook und Mark Everist. Oxford, New York: Oxford University Press, S. 378-402.

Fohrmann, Jürgen (1997). »Diskurstheorie(n). «In: Reallexikon der deutschen Literaturwissenschaft. Neubearbeitung des Reallexikons der deutschen Literaturgeschichte. Hg. v. Klaus Weimar gemeinsam mit Harald Fricke, Klaus Grubmüller und Jan-Dirk Müller. Berlin, New York: Walter de Gruyter, S. 372-74.

Foucault, Michel (1973). Archäologie des Wissens. Frankfurt/M.: Suhrkamp.

Jäger, Siegfried (2006). »Diskurs und Wissen: Theoretische und methodische Aspekte einer kritischen Diskurs- und Dispositivanalyse.« In: Handbuch Sozialwissenschaftliche Diskursanalyse 1. Theorien und Methoden. Hg. v. Reiner Keller, Andreas Hirseland und Werner Schneider. Wiesbaden: VS Verlag (2., aktualisierte und erw. Aufl.), S. 83-114.

Jauß, Hans Robert (1979). "Literaturgeschichte als Provokation der Literaturwissenschaft [1967].«In: Ders., Literaturgeschichte als Provokation. Frankfurt/M.: Suhrkamp, S. 144-208.

Kahn-Harris, Keith (2007). Extreme Metal: Music and Culture on the Edge. Oxford, New York: Berg.

Kerman, Joseph (1983). »A Few Canonic Variations.«In: Critical Inquiry 10, S. 10726.

Markowitsch, Hans J. / Welzer, Harald (2005). Das autobiographische Gedächtnis. Hirnorganische Grundlagen und biosoziale Entwicklung. Stuttgart: Klett-Cotta.

Popoff, Martin (2003). The Top 500 Metal Songs Of All Time. Toronto: ECW Press.

Rock Hard (Hg.) (2005). Best of Rock \& Metal - die 500 stärksten Scheiben aller Zeiten. Königswinter: Heel.

Schäfer, Frank (2001). Heavy Metal. Geschichten, Bands und Platten. Leipzig: Reclam.

Tulving, Endel (2005). »Das episodische Gedächtnis: Vom Geist zum Gehirn.«In: Warum Menschen sich erinnern können. Fortschritte der interdisziplinären Gedächtnisforschung. Hg. v. Harald Welzer und Hans J. Markowitsch. Stuttgart: Klett-Cotta, S. 50-77.

Udo, Tommy (2002). Brave Nu World. London: Sanctuary.

Vansina, Jan (1985). Oral Tradition as History. Madison: University of Wisconsin Press.

Walser, Robert (1993). Running With The Devil - Power, Gender and Madness in Heavy Metal Music. Hanover, London: Wesleyan University Press.

Webber, William (1999). "The History of Musical Canon. "In: Rethinking Music. Hg. v. Nicholas Cook und Mark Everist. Oxford, New York: Oxford University Press, S. 336-355.

Weinstein, Deena (1991). Heavy Metal: A Cultural Sociology. New York: Lexington.

Welzer, Harald (Hg.) (2001). Das soziale Gedächtnis. Geschichte, Erinnerung, Tradierung. Hamburg: Hamburger Edition.

Welzer, Harald (2002). Das kommunikative Gedächtnis. Eine Theorie der Erinnerung. München: Beck.

Welzer, Harald (2005). „Über Engramme und Exogramme. Die Sozialität des autobiographischen Gedächtnisses. « In: Warum Menschen sich erinnern können. 
Fortschritte der interdisziplinären Gedächtnisforschung. Hg. v. Harald Welzer und Hans J. Markowitsch. Stuttgart: Klett-Cotta, S. 111-128.

\title{
Diskographie
}

AC/DC (1980). Back In Black. Atco 16018.

Black Sabbath (1970). »Paranoid. « Auf: Paranoid. Vertigo 6360011.

Celtic Frost (1984). Morbid Tales. Noise Records N 0017.

Celtic Frost (1985). To Mega Therion. Noise Records N 0031.

Cindy \& Bert (1971). "Der Hund von Baskerville«. Cornet 3220; wiederveröffentlicht auf: V.A. (2005). Beatschuppen. Panatomic PALP 004.

Death (1987). Scream Bloody Gore. Under One Flag Flag 12.

Emperor (1994). In The Nightside Eclipse. Candlelight Records Candle008CD.

Entombed (1990). Left Hand Path. Earache Records MOSH 21.

Guns N' Roses (1987). Appetite For Destruction. Geffen 24148.

Iron Maiden (1982). "The Number Of The Beast. « Auf: The Number Of The Beast.

EMI 1C 064-07608.

Led Zeppelin (1971). IV. Atlantic Records 756782638.

Mayhem (1994). De Mysteriis Dom Satanas. Deathlike Silence Records DSP 6.

Metallica (1983). Kill 'em All. Music For Nations MFN7.

Metallica (1984). Ride The Lightning. Music For Nations MFN 27.

Metallica (1986). Master Of Puppets. Music For Nations MFN 060.

Morbid Angel (1989). Altars Of Madness. Earache Records MOSH 11.

Napalm Death (1987). Scum. Earache Records MOSH 3.

Possessed (1985). Seven Churches. Roadrunner Records 9757.

Queensrÿche (1988). Operation: Mindcrime. EMI-Manhattan Records 7486401.

Sepultura (1989). Beneath The Remains. Roadracer Records 9511.

Slayer (1986). Reign In Blood. DefJam Recordings GHS 24131.

\begin{abstract}
Scholarly discussion of cultural memory knows both concepts: canon and stream of tradition. It is argued that in the case of popular music the concept of stream of tradition should be preferred to the concept of canonisation because of its more open character and the short lifespan of popular music. How to gain access to the stream of tradition of a musical style is exemplified by a sample of shortlists of 'The best Heavy Metal ... of all time--type. Conclusions of the evaluation, which is part of the author's ongoing doctoral thesis, show that the stream of tradition appreciates neither bands nor single albums but creative periods.
\end{abstract}

